\title{
Obstetric training: competence and care in birth assistance
}

This thesis consists of an analysis of obstetric training during medical school. It is based on ethnographic research undertaken at two acknowledged medical schools in São Paulo, Brazil. The objective was to study how technical and scientific competence and care, that is, the relationship with the patient, are articulated in theoretical and practical training of birth assistance. The techniques employed in fieldwork were: participant observation, semi-structured interviews and, in a complementary form, the analysis of textbooks and assistance protocols. A characterization of the curriculum offered by the schools and an examination of students experience with respect to learning the theory and practice of obstetrics, including the supervision of the various activities involving obstetric assistance are discussed. Description and analysis of the scission between the development of technical and scientific competence in the educational process was undertaken. The implications of this scission for training in the practice of medicine as a moral dependent technique are discussed. Great emphasis is placed on the development of scientific knowledge during training. Even in the clinical years, emphasis is placed on learning to construct clinical narratives, placing priority on memory and the oral transmission of knowledge rather than written registration and consultation of patience's charts. Learning and training is based in part on so-called "classical" concepts, which sustain a pathological approach to birthing. In training, this approach involves norms of conduct that have been questioned by scientific evidence and that have even been abandoned in other contexts. Obstetrical decisions involving conduct and training are not taken in conjunction with the women receiving assistance who frequently are not even consulted or informed of these decisions. Sometimes prejudices influence medical judgment and decision-making. There are few parameters to evaluate student attitudes in their interaction with patients. In the work stations where students interact more with patients there is less supervision. Informal arrangements between assistants with respect to rounds conflict with the formal schedules of the obstetric services associated to the medical schools where research was undertaken. These arrangements subordinate the institutional objectives of good medical practice and assistance to the individual and collective interests of the obstetricians responsible for supervising training. These arrangements are a component of the hidden curriculum and serve as a model for other arrangements involving residents and/or students. Throughout the obstetric training of medical students, interactions between the subjects involved in the medical act contribute in several ways to disqualify the medical practice of obstetrics as a morallydependent technique.

Sonia Nussenzweig Hotimsky Tese (Doutorado), 2007 Departamento de Medicina Preventiva, Faculdade de Medicina, Universidade de São Paulo, SP. sonianhotimsky@uol.com.br

Full text:

http://www.teses.usp.br/teses/disponiveis/5/

5137/tde-14112007-082030/ 